\title{
Voice Loops as Cooperative Aids in Space Shuttle Mission Control
}

\author{
Jennifer C. Watts, David D. Woods, James M. Corban, Emily S. Patterson \\ Cognitive Systems Engineering Laboratory \\ The Ohio State University \\ watts@csel.eng.ohio-state.edu
}

\author{
Ronald L. Kerr, LaDessa C. Hicks \\ NASA Johnson Space Center \\ Houston, Texas
}

\begin{abstract}
In domains like air traffic management, aircraft carrier operations, and space mission control, practitioners coordinate their activities through voice loops that allow communication among groups of people who are spatially separate. Voice loops have evolved into essential coordination support tools for experienced practitioners in space shuttle mission control, as well as other domains. We describe how voice loops support the coordination of activities and cognitive processes in event-driven domains like space shuttle mission control. We discuss how the loops help flight controllers synchronize their activities and integrate information, and how they facilitate directed communication and support the negotiation of interruptions. In addition, we suggest factors like attentional cues, implicit protocols, and the structure and features of the loops, which might govern the success of voice loops in the mission control domain. Our results should provide insight into the important functions that should be considered in the development of systems intended to support cooperative work.
\end{abstract}

\section{KEYWORDS}

voice loops, space shuttle mission control, control rooms, coordination, ethnography

\section{INTRODUCTION}

In event-driven domains like nuclear power and space mission control, cognitive activities such as monitoring and anomaly response are distributed across interdependent sets of practitioners. These practitioners must coordinate their activities to accomplish everyday tasks and goals and to handle the cascade of disturbances produced by faults [12]. As Hughes \& Shapiro [6] and Bentley et al [1] note, practitioners in these kinds of domains must be able to coordinate their efforts on a "moment to moment basis, in response to constantly changing circumstances [6]."

Permission to make digital/hard copies of all or part of this material for personal or classroom use is granted without fee provided that the copies are not made or distributed for profit or commercial advantage, the copyright notice, the title of the publication and its date appear, and notice is given that copyright is by permission of the ACM, Inc. To copy otherwise, to republish, to post on servers or to redistribute to lists, requires specific permission and/or fee.

Computer Supported Cooperative Work '96, Cambridge MA USA

(c) 1996 ACM 0-89791-765-0/96/11 ..\$3.50
In some of these domains, including air traffic management, aircraft carrier operations [11] and space mission control, this coordination is supported by voice loops, which allow communication among groups of people who are spatially separate.

This paper describes how voice loops support the coordination of activities and cognitive processes in space shuttle mission control. To outsiders listening in, the communication occurring on these loops may seem noisy and confusing. However, voice loops are essential coordination support tools for experienced practitioners in shuttle mission control, as well as other domains [11]. We analyze how voice loops support complex coordinated activity in event-driven domains and suggest reasons for why voice loops have been successful.

\section{METHODS}

Our results are based on ethnographic observations of how flight controllers use voice loops to successfully coordinate their activities during space shuttle operations. Two of the authors (LH and RK) are actual flight controllers, and the remaining authors observed mission control operations during a total of 27 flight control simulations held as preparation for upcoming missions or for flight control training purposes (over 130 hours of observation). These simulated missions include a full complement of astronauts and flight controllers supporting each flight control console. Simulated missions are held in the actual facilities used to support shuttle operations and include multiple failures. A simulated mission lasts between 3 to 5 hours; longer simulations continue for several days. We also observed mission control operations during portions of four different real-time shuttle missions.

In addition, during low-intensity portions of the missions and training simulations that we observed, we conducted interviews with flight controllers discussing how voice loops support coordination across flight controllers. The controllers described formal and informal protocols and procedures that govern the usage of voice loops in this environment. For example, they described which loops 
they monitor, why they monitor them, and how and when they are expected to speak on these loops.

\section{COOPERATIVE STRUCTURE OF SPACE SHUTTLE MISSION CONTROL}

\section{Hierarchical structure}

NASA's Mission Control Center at the Johnson Space Center in Houston, Texas, is the nerve center for all space

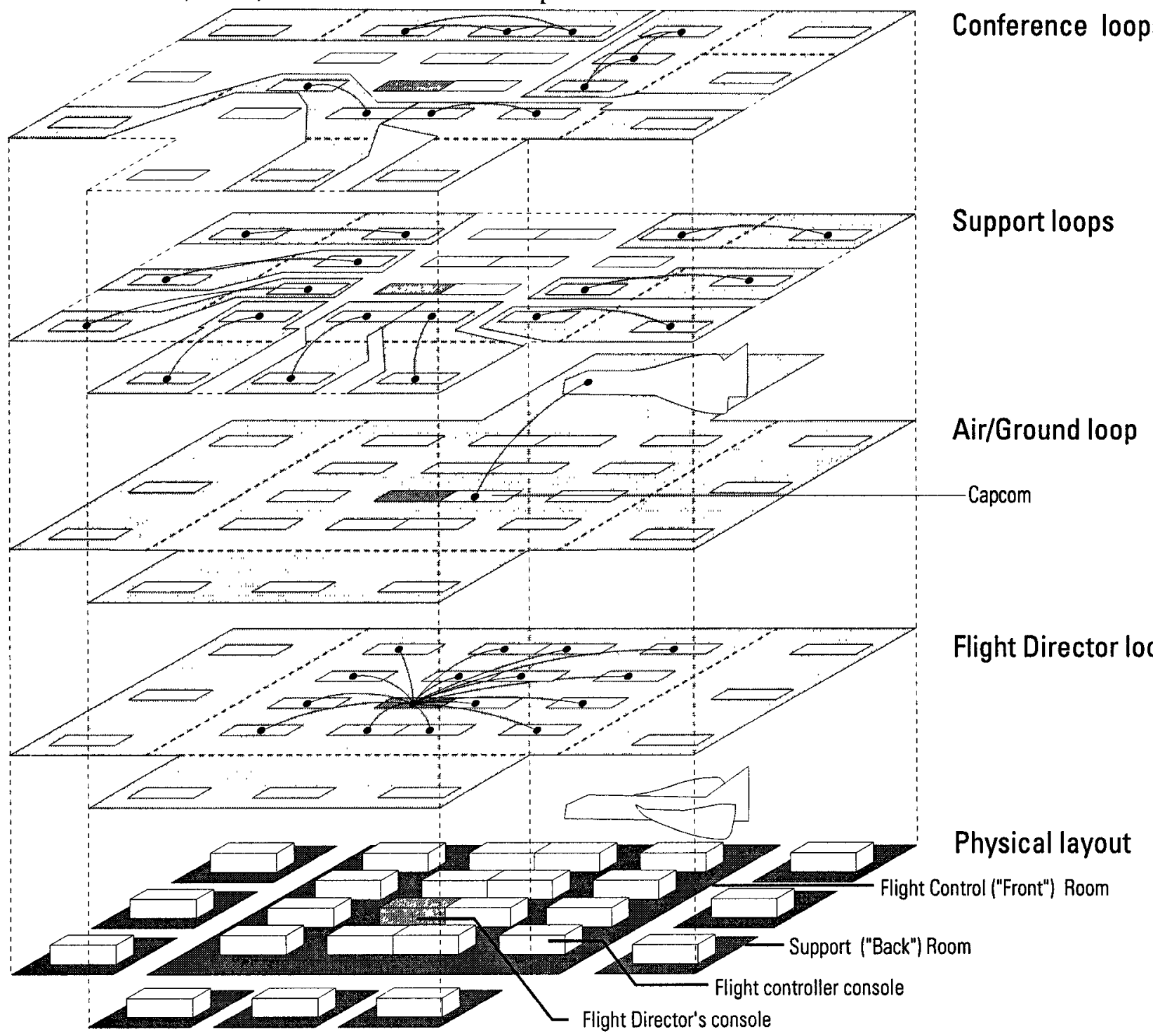

(C) Corban, Patterson, Woods and Kidwell

\section{Legend}

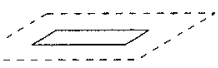

No shading around a console indicates that that controller does not listen to or speak on that loop. shuttle flights [10]. Teams of engineers and technicians monitor spacecraft systems and activities 24 hours a day during missions. They monitor the astronauts' activities, track system parameters to be sure missions are proceeding as expected, and provide expertise to deal with the unexpected. These teams are arranged in a hierarchical structure which enables efficient coordination of processes and activities during shuttle missions (Figure 1).

Conference loops

Support loops

Air/Ground loop

Capcom

Flight Director loop

Shading around a console indicates that the controller normally listens to that loop. but is not permitted to speak.

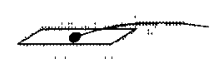

A black dot on a console indicates that the controller is permitted to speak on that loop. The line indicates who she speaks to.

Figure 1. Structure of the voice loops 
Directly supporting the flight director is a team of approximately twenty flight controllers. Each of these controllers is responsible for monitoring specific shuttle subsystems. For example, one controller is responsible for the electrical subsystems and another controller is responsible for the mechanical subsystems. The flight director and his supporting flight controllers are co-located in the Flight Control Room--what is referred to as the front room. This is the control room that is shown on NASA Select TV. Almost everyone has seen the television or movie pictures of flight controllers working at their consoles, headsets in place (Figure 2).

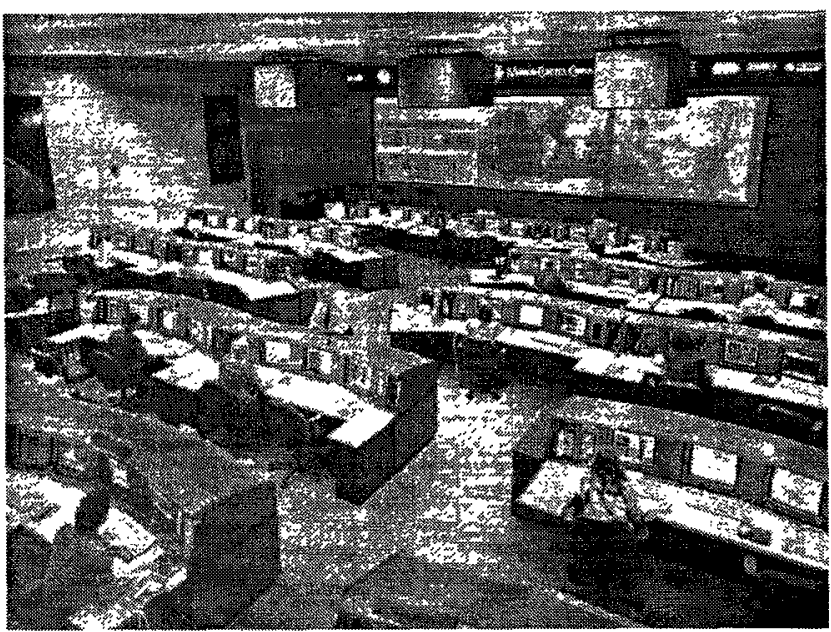

Figure 2. The front room in the Mission Control Center

In addition to reporting to the flight director, each supporting flight controller is also a supervisor of a separate staff of flight controllers and engineers. This supporting staff is located in separate facilities that are commonly called the back rooms. The back rooms consist of a primary support staff who maintain continual contact with front room controllers, as well as a secondary support staff who provide extra information about shuttle subsystems in the event of anomalies. The practitioners in the back rooms help the main flight controllers monitor their subsystems during nominal operations, and also aid in diagnosis and re-planning processes when anomalies occur.

\section{Scopes of responsibility}

Members of the various hierarchical levels in shuttle mission control possess different scopes of authority and responsibility. The flight director is ultimately responsible for decisions related to the operations of the shuttle. He tracks events and coordinates shuttle activities to ensure that mission goals are accomplished. Therefore, his scope of responsibility is very broad.

The main flight controllers who support the director possess a medium-range scope of responsibility which requires them to track nominal events and respond to any off nominal events that may occur in their specific subsystems. The controllers view their systems through computer screens containing telemetry data transferred from sensors on the shuttle. Each controller monitors custom screens containing telemetry data representing the specific systems within their scope of responsibility. For example, the electrical controller monitors screens containing telemetry data coming from all of the electrical systems.

Controllers integrate the telemetry data on the screens in order to extract significant events occurring within the subsystems. For example, the mechanical systems controller may compare temperature and pressure values of a fuel line to determine whether the line has become blocked or frozen. Based on events extracted from the data, controllers initiate actions to maintain system stability (e.g., if the mechanical systems controller notices that the fuel line has become too cold, she may ask that the astronauts turn on a line heater).

Controllers normally display telemetry data on their consoles from systems within their own scope of responsibility. Therefore, they do not have visual access to data and events occurring in other subsystems. They do have the ability to access telemetry displays from systems outside their immediate scope of responsibility. However, there are no visual cues to tell them whether information in other displays is relevant to their tasks and goals. Even so, it is often necessary for flight controllers to be aware of the events and activities which occur in related subsystems. This awareness allows them to coordinate their activities within the general context of mission operations [3]. Coordination across shuttle subsystems is essential because many of the subsystems are interconnected. Often, events in one subsystem impact other subsystems; for example, if a controller is aware of an unexpected event in a related subsystem, she can anticipate how that event will affect her own subsystems.

The support staff in the back rooms focus more deeply on their specific subsystems. They have the responsibility to analyze patterns, diagnose faults, and assess the consequences of faults for mission operations. The results of their analyses are passed to the front room and ultimately to the flight director as input to decisions. These back room controllers not only monitor the same displays as the front room controllers, but they also have access to more detailed information about the subsystems. For example, the front mechanical systems controller has access to telemetry displays which are updated every second. In addition to these displays, the back room controllers also have access to paper strip charts which are updated much more frequently than the raw data displays. Therefore, the back room controllers are able to pick up subtle trends that may not be evident to the front room controller.

\section{COMMUNICATION IN MISSION CONTROL}

In order to facilitate coordination across shuttle subsystems, the system of flight controllers communicates via the audio system called voice loops. They use the loops to communicate and to monitor conversations discussing events and activities that occur throughout shuttle operations. 
The structure of the voice loops reflects the structure of mission control and provides common links between functionally related groups who must coordinate their activities and processes in the current mission context. Instead of being organized around one global loop on which practitioners must take turns speaking, NASA has devised a structure of multiple loops which are monitored in parallel. Rather than monitor one conversation at a time, each controller monitors several loops on which conversations can occur in parallel. Therefore, the voice loops act as a communication mechanism providing auditory connections between people and groups who must coordinate their activities.

\section{Kinds of loops}

Each controller typically monitors a minimum of four loops: the flight director loop, the air-to-ground loop, the front-to-back loop, and a conference loop (see Figure 3).

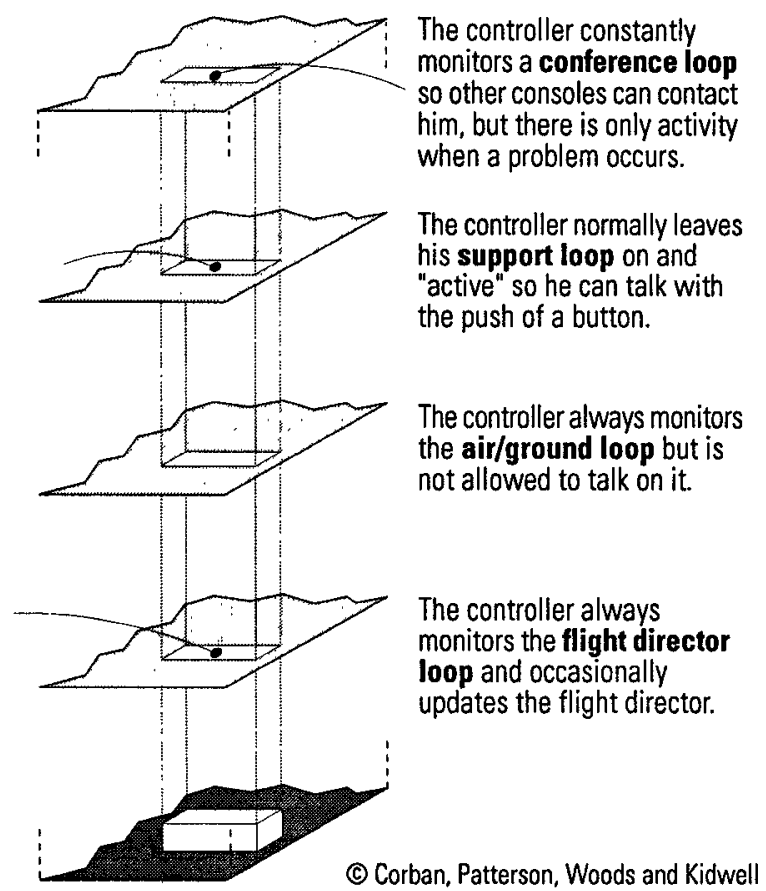

Figure 3. A front room controller's voice loop system

\section{The Flight Director loop}

The flight director loop is arguably the most significant loop in the system. It is monitored by all flight controllers and many of the support teams. It consists primarily of communication between the flight director and the main front room controllers. Controllers report and discuss relevant issues with the flight director on this loop, with the intention not only of informing the director, but of conveying their situation to the rest of Mission Control. This is also the loop where the flight director broadcasts announcements concerning major decisions, changes to the mission plan, or clarifications. Because of the importance of this loop, only issues of high significance are discussed here, and communication is kept clear and concisc.

\section{Air to Ground loop}

The air to ground loop consists of communication between the astronauts on the shuttle and the flight controller called "CAPCOM". All front room controllers, and nearly everyone in the back support rooms monitor the Air to Ground loop. However, to avoid unnecessary interruptions of astronaut activity, CAPCOM is the only ground controller who speaks on this loop. CAPCOM is usually an astronaut himself, and sits next to the flight director in the flight control room. Communication between the Flight Director and CAPCOM concerning what to say on the loop can either take place on the Flight Director loop or face-to-face.

Front-to-Back (support) loops

In addition to the Flight Director loop and the Air to Ground loops, a separate front-to-back loop exists for each main controller in the front room. On these loops, the main front room controller discusses events and activities with support staff who are located in the back rooms. Discussions on these loops are much more detailed and less formal than discussions on the flight director loop. Here, controllers may discuss events and telemetry values at length. For example, if a controller notices an abnormal telemetry value, she can discuss it with her back room controller to determine the cause and any actions that must be taken.

\section{Conference loops}

The fourth kind of voice loop in shuttle mission control is typically referred to as a conference loop. These loops allow ad-hoc groups to form when relevant events arise. They allow custom groups of controllers whose functions overlap to discuss common issues and coordinate activities. Conference loops often lie unused until a need arises for them. However, they are always on, and controllers are always connected to them so that related controllers can contact them when necessary. Sometimes during periods of heavy activity, these loops may also be monitored by controllers who may not be directly involved in the discussions that occur.

While it may seem difficult to monitor multiple loops in parallel, this ability is essential to controllers' activities and goals. For example, during our observations, the mechanical systems controller noticed an abrupt change in the data on her telemetry screens. In order to determine the cause of this change, she monitored and interacted with four loops in parallel. She monitored the air to ground loop to discover whether the astronauts were experiencing any abnormal circumstances aboard the shuttle and listened to the flight director loop to discover whether other controllers were also seeing strange data patterns. She also contacted a related controller on the conference loop to give him a "heads up" that her systems were functioning abnormally, and she discussed the details of the data with her back room staff to determine what might have caused the abnormality. Eventually, she heard the electrical controller inform the flight director that an electrical bus had failed. This failure could logically account for the unexpected changes in her system data. She contacted the electrical controller on the 
conference loop to find out whether the bus would be fixed, and discussed the impact of this failure with her support staff over the front-to-back loops.

\section{HOW VOICE LOOPS SUPPORT COORDINATION}

One might think that voice loops are used primarily for directed communication, where one controller uses the voice loops to speak directly to another controller. Although this kind of communication is supported by voice loop technology, the most important function of the voice loops in mission control is that they afford the ability to listen in to events and activities that occur across mission processes (e.g. [13]). The ability to listen in to discussions on the loops allows controllers to pick up relevant events and activities without disrupting their ongoing work within the scope of their responsibility.

Similar kinds of coordination have also been observed in the control rooms of other event-driven domains. Luff, Heath, and Greatbach [8] noticed it in Line Control Rooms in the London Underground, where underground controllers have developed the technique of talking out loud to themselves about changes that they make in schedules during crisis situations. When controllers express changes out loud, other controllers in the vicinity can take note of important changes which will affect their own schedules without having to interrupt the busy controller during the crisis situation. Similar coordination also occurs in the domain of aircraft carrier operations, Rochlin notes: “... everyone involved ... is part of a constant loop of conversation and verification taking place over several different channels at once. At first little of this chatter seems coherent, let alone substantive, to the outside observer. ... one discovers that seasoned personnel do not 'listen' so much as monitor for deviations, reacting to almost anything that does not fit their expectations ...[11]."

Voice loops help controllers remain aware of shuttle events and activities as they focus on their immediate tasks and goals. Dourish and Bellotti [3] refer to this function as a passive awareness mechanism and Woods [13] calls it preattentive reference. The auditory messages transmitted via voice loops allow flight controllers to shift their attention and activities dynamically and opportunistically in response to the changing state of affairs within the shuttle systems (e.g. [3]). The loops allow practitioners the opportunity to peripherally monitor other teams' activities so that a team of flight controllers, even when working independently, is both communicating their activities and providing other teams with the opportunity to observe consequences for their own actions (as in [13] and [3]).

An important source of information about the events and activities occurring across shuttle operations is the flight director loop. If a controller informs the flight director about an unexpected event on his loop, he is also informing all of the other flight controllers. Also, the flight director can broadcast messages to the community without selecting who must receive the message. When he broadcasts the message, relevant controllers can focus their attention on the message, and other controllers can filter the message. This characteristic makes the system more robust because it eliminates the opportunity for the director to unintentionally leave someone "out of the loop".

Voice loops facilitate coordination across controllers by facilitating group synchronization during nominal and offnominal events. They aid controllers' tasks and activities by integrating different levels and kinds of information. In addition, they facilitate communication. They contribute to the common ground between controllers by creating a common frame of reference ([2], [7]) and they mediate the timing of communication by allowing controllers to judge the interruptability of co-workers.

\section{SYNCHRONIZATION}

When flight controllers are aware of important mission events and activities, they can anticipate problems in their systems and prepare for future actions which will be required. Anticipation has also been found to be important in control rooms of other domains in which teams coordinate to handle high-tempo, uncertain situations [6]. Anticipation is important because it allows controllers to synchronize their communication and actions over time. For example, if a failure occurs in a subsystem, the flight director will ask related subsystem controllers about the impacts of that failure on their systems. When controllers hear about the failure on the flight director's loop, they can anticipate questions from the director, and prepare to answer them without delay. Controllers can also anticipate actions that will be required for them. For example, an anomaly in one subsystem might require diagnostic tests in another system. When the controller hears about the anomaly on the voice loops, she can anticipate the requirement of these tests, and prepare to conduct them when they are necessary.

One way voice loops aid synchronization is by affording the ability to track the tempo of mission processes. Since shuttle systems are interconnected, a failure in one subsystem may cause a cascade of disturbances throughout related systems. This cascade of disturbances causes a cascade of activity as controllers respond to these disturbances. For example, if an event like a complex anomaly occurs in a shuttle subsystem, the event triggers diagnostic activity in all related subsystem teams. This activity generates more communication across teams over the voice loops. Therefore, it is possible for controllers to track the cascade of disturbances in shuttle systems by tracking the cascade of activities that occur in response to these disturbances. This general indication of activity tempo allows controllers to synchronize their processes and activities with rest of the flight control team.

\section{INTEGRATING INFORMATION}

A characteristic of voice loops which allows them to successfully support coordination of activities and processes in shuttle mission control is that their hierarchical structure facilitates the economical integration of information at different levels of abstraction. Also, the loops integrate different kinds of information. This integrated information 
provides an indication of the tempo of events and activities in the mission process.

\section{Integrating levels of information}

The hierarchical structure of the voice loops allows controllers to transmit an overview of mission events and activities in parallel with detailed information relevant to flight controllers' subsystems. Since the flight director must maintain a global understanding of critical space shuttle events and activities, and since all controllers monitor his loop, information on the loop usually consists of a brief summary of detailed events related to a subsystem, combined with a recommendation for action. For example, if a fuel line freezes, controllers must infer this event from temperature and pressure data on their display screens. However, instead of describing the temperature and pressure of the line on the flight director loop, controllers tell the flight director that a line is frozen, and make a recommendation for future actions. Meanwhile, controllers discuss the frozen line in much more detail on the front to back loops. Therefore, the flight director loop functions as an overview of events and activities. This overview loop can be monitored in parallel with front to back loops, which discuss events at greater levels of detail.

\section{Integrating kinds of information}

In addition to integrating levels of information, voice loops also integrate different kinds of information. Two of the most important kinds of information integrated in the voice loops are information about shuttle events and information about the activities related to these events.

\section{Information about mission events \\ A general tendency in computer support systems is to provide raw data about the monitored process. For example, the mission control display screens may provide information about a system's temperature and pressure. The controllers must integrate this information to determine the system's global status and behavior. The voice loops, however, allow controllers to take advantage of the data integration performed by other controllers. Instead of passing raw data about related systems from one controller to another, voice loops pass integrated, event- level information between controllers monitoring interconnected systems.}

The ability to pass event-level information is essential to shuttle controllers' tasks and goals. As mentioned above, anomalies occurring in one system often cause a cascade of disturbances through other systems. Therefore, in order for controllers to meet the goal of anticipating and preparing for potential problems in their subsystems, they must be aware of events that are happening in related subsystems. The voice loops facilitate this awareness by passing eventlevel information between controllers of related subsystems.

\section{Information about mission activities}

Research has shown that practitioners in process control domains must not only keep track of the monitored process, but they must also track the activities of other agents who are affecting that monitored process ([12]; [7]). This principle applies in the shuttle mission control domain as well. For example, if the electrical controller performs a test of an electrical bus, or intentionally removes a bus from service, his actions will affect the subsystems that receive power from that bus. Therefore, the controllers of these subsystems must be aware of the electrical controller's actions so they will expect to see the impact of the electrical test on their subsystems.

Voice loops provide an elegant view into the processes and activities of other practitioners. By monitoring discussions on the loops, controllers can pick up conversations about activities performed by other controllers which will eventually impact their subsystems. In addition to activities of co-workers which will impact their subsystems, controllers can also detect discussions about events which will require them to initiate their own activities. For example, when the electrical controller tells the flight director that he will be performing a test, the flight director may commonly respond to this information by asking relevant controllers how the test will impact their subsystems. Therefore, when one of these controllers hears the message from the electrical controller on the flight director loop, she can anticipate a question from the flight director, and prepare to answer the question without delay.

The ability to track activities over the voice loops also allows controllers to pick up information about the status of processes and activities without interrupting the controllers involved. If an anomaly in the mechanical systems occurs, the flight director can monitor the front-toback loops of the mechanical console to track the status of the diagnosis or response process. This way, he can track the line of reasoning in the mechanical controllers' processes without interrupting their activities by asking for an update on their status.

\section{FACILITATING COMMUNICATION}

When controllers listen to discussions of mission events and activities on the voice loops, they build a common ground with other controllers. This common ground facilitates directed communication and helps controllers negotiate interruptions.

\section{Facilitating directed communication}

Since controllers are spatially separated, they could experience problems keeping all related controllers "in the loop", and aware of their activities. For example, controllers could use an asynchronous communication mechanism like email instead of voice loops. In this case, if an unexpected event occurred in a controller's subsystems, he would have to remember who needed to hear about the event, find the email addresses of these people, and send a message to them. He would then have to wait for them to read their email and respond to his message. 
The structure of voice loops eliminates many of the problems that might occur with an asynchronous system. Instead of forcing controllers to remember which individuals should be notified of an unexpected event, voice loops allow them to speak into a loop that is being monitored by the appropriate groups. This structure also eliminates the extra work required to find addresses of appropriate individuals, and allows an immediate response from members of the group.

\section{Negotiating interruptions}

The information that voice loops provide about the tempo and activities of controllers helps co-workers negotiate interruptions. Since controllers are aware of the tempo of mission processes, they can gain insight into co-workers' workload. If a controller decides to communicate with a coworker, he can listen to that person's loop to determine her workload before he contacts her. By doing this, he can determine whether she is too busy to discuss the issue of interest, and whether his issue is as critical as other issues that the co-worker is addressing. Therefore, the co-worker does not have to direct her attention away from current tasks and goals to decide whether new messages are critical. This characteristic helps controllers focus on the most critical issues during periods of high workload.

\section{COPING WITH VOICE LOOP CACOPHONY}

How can controllers extract meaningful information from the noisy, multi-party exchange occurring on the voice loops? While we cannot explain the detailed cognitive processes that allow this to happen, the successful history of the voice loop system in mission control demonstrates that practitioners are able to extract meaningful information from these loops. The controllers possess the competence to extract important cues that are relevant to their goals and activities as they focus their attention on the systems within their scope of responsibility.

Based on our observations, combined with previous laboratory studies of attention ([5], [4]), we can suggest two characteristics which probably contribute to the success of voice loops as coordinative aids. One characteristic which probably contributes to the success of voice loops as a coordinative tool is the combination of internal and external cues which serve to direct practitioners' attention to relevant events and activities within their context. During our interviews, many controllers mentioned that external cues like voice tone and quality, as well as key words and phrases, helped them direct their attention to the events and activities of related controllers. In addition to these external cues, controllers mentioned that they also direct their attention based on internal cues, including their expectations and assessment of their situation. For example, one controller said that if an anomaly occurs within his subsystems, this event primes him to pick up key words and phrases related to this anomaly in the voice loop discussions.

Another characteristic that probably contributes to the success of voice loops in shuttle mission control is the implicit protocols, as well as the structures and features of the loops, which have evolved in the mission control domain.

\section{Implicit protocols}

Controllers make use of several implicit protocols which probably contribute to their ability to extract and communicate meaningful information across the loops. These protocols include protocols governing which loops are monitored, the response on demand principle, and highly coded language.

One of the most important protocols contributing to the success of voice loops is the implicit protocol that governs which loops are monitored by the controllers. As we mentioned above, each controller monitors the flight director loop in parallel with his front-to-back loops. Therefore, the loops serve as an overview of general shuttle events in parallel with detailed information about the system within each controller's scope of responsibility.

Another protocol is the response on demand principle. This principle says that controllers should respond to other controller's directed messages immediately, correctly, and precisely. This kind of communication reduces extra noise on the loops, and minimizes opportunities for controllers to pass inaccurate information over the loops.

A third implicit protocol that reduces extra noise on the loops is the use of highly coded language. Controllers use abbreviations and acronyms which carry lots of information, but do not take up too much bandwidth. The following example illustrates how this protocol was used during ascent, which is a high-tempo period. The mechanical systems controller (MMACS) informs the flight director (flight) on the flight director loop that there is a leak in a hydraulic system after requesting for permission to speak by stating who he is calling (flight) and who he is (MMACS). This communication is heard by everyone in mission control since they all monitor the flight director loop; at this point, they start checking their back rooms to see how the leak will impact their subsystems. On a different loop, another controller (FDO) is broadcasting landmark events on the ascent timeline such as the main engine cut-off (MECO). In this example, this landmark is particularly important because one concern is if the leaking system will support ascent until MECO.

$\begin{array}{ll}\text { [MMACS] } & \text { Flight, MMACS } \\ \text { [Flight] } & \text { Go, MMACS } \\ \text { [FDO] } & \text { MECO } \\ \text { [MMACS] } & \text { We're looking at what may be a } \\ \text { [FDO] } & \text { Press-to-MECO } \\ \text { [MMACS, simultaneous with FDO] small hydraulic leak } \\ \text { [Flight] } & \text { Go ahead, MMACS. Say it again. } \\ \text { [MMACS] } & \text { A small hydraulic leak on hydraulic } \\ \text { system 3. Looks like it will make MECO. }\end{array}$




\section{Structure and features of the loops}

Another feature of the loops which probably contributes to their success is the opportunity to listen to different loops at different volume levels. Controllers can segregate loops by listening to them at different volumes. Therefore, they can monitor loops which are busy or less relevant to their tasks and goals at a lower volume than loops which provide more relevant information.

The voice loop structure also provides the opportunity to tailor the set of loops that is being monitored. If controllers find that a conference loop is not relevant to their tasks and goals, they can stop monitoring it. On the same note, if controllers realize that important events are being discussed on another loop, they can tune into that loop while the events are being discussed.

A final feature of the loops which contributes to voice loop success in the mission control domain is the structure of the multiple loops. Since the loops are divided into functionally separate loops, controllers are not forced to monitor all communication that occurs over all loops. Instead, they can choose to monitor only the loops that are relevant to their tasks and goals, and therefore minimize unnecessary noise on their loops.

\section{CONCLUSION}

Our studies of voice loops in space shuttle mission control, combined with the successful history of the loops, demonstrate that controllers are able to extract meaningful patterns from the noisy, multi-channel loops, even during periods of high workload and activity. These loops help controllers synchronize their activities and provide access to integrated kinds and levels of information about mission events and activities. They also facilitate directed communication and help controllers negotiate interruptions.

Some of the factors which seem to make voice loops successful include the implicit protocols which govern their use, the structure and features of the loops, and the mix of external and internal cues which arise through their use to help controllers direct their attention to relevant information about shuttle behavior, as well as the activities of other controllers. A better understanding of how voice loops have evolved to support coordination in space shuttle mission control should provide insight into the important functions that should be considered in the development of systems intended to support cooperative work in similar event-driven domains.

\section{ACKNOWLEDGMENTS}

Research support was provided by NASA Johnson Space Center under Grant NAG9-390, Dr. Jane Malin technical monitor. We are particularly grateful to the flight controllers who shared their expertise with us and allowed us to observe their operations during training simulations and actual missions. Additional support was provided by two National Science Foundation (NSF) Graduate Fellowships. Any opinions, conclusions, or recommendations expressed in this publication are those of the authors and do not necessarily reflect the views of NSF.

\section{REFERENCES}

1. Bentley, R., Hughes, J. A., Randall, D., Rodden, T., Sawyer, P., Shapiro, D., \& Sommerville, I. (1992). Ethnographically-informed systems design for air traffic control. In 1. ACM (Ed.), Proceedings of Computer Supported Cooperative Work.

2. Clark, H., \& Brennan, S. (1991). Grounding in Communication. In L. R. a. S. Teasley (Eds.), Socially Shared Cognition Washington, DC: American Psychological Association.

3. Dourish, P., \& Bellotti, V. (1992). Awareness and coordination in shared workspaces. In Proceedings of the CSCW'92 Computer supported Cooperative Work. Toronto, Canada:

4. Folk, C. L., Remington, R. W. and Johnston, J. C. 1992, Involuntary covert orienting is contingent on attentional control settings. Journal of Experimental Psychology, Human Perception and Performance, $18,1030-1044$.

5. Gopher, D. 1991, The skill of attention control, Acquisition and execution of attention strategies, in Attention and Performance XIV (Lawrence Erlbaum Associates, Hillsdale, NJ).

6. Hughes, J. A., \& Shapiro, D. (1992). Faltering from ethnography to design. In 1. ACM (Ed.), Proceedings of Computer-Supported Cooperative Work.

7. Johannesen, L., Cook, R., \& Woods, D. (1994). Grounding explanations in evolving diagnostic situations CSEL Report 1994-TR-03). The Ohio State University, Cognitive Systems Engineering Laboratory.

8. Luff, P., Heath, C., \& Greatbatch, D. (1992). Tasksin-interaction: Paper and screen based documentation in collaborative activity. In Proceedings of CSCW'92 Computer-Supported Cooperative Work. .

9. Murray, C. and Cox, C. B. 1989, Apollo, The Race to the Moon (Simon \& Schuster, New York).

10. NASA - The Shuttle News Reference Manual, found on the World-wide web. URL: HTTP://www.ksc.nasa.gov/shuttle/technology/stsnewsref/sts-jsc.html\#sts-jsc-mcc

11. Rochlin, G. I., La Porte, T. R. and Roberts, K. H. 1987, The self-designing high-reliability organization, 
Aircraft carrier flight operations at sea. Naval War College Review,, Autumn, 76-90.

12. Woods, D. D. 1994, Cognitive Demands and Activities in Dynamic Fault Management, Abduction and Disturbance Management, in N. Stanton (ed.)., Human Factors of Alarm Design (Taylor \& Francis, New York).

13. Woods, D.D. 1995, The alarm problem and directed attention in dynamic fault management. Ergonomics, 38(11), 2371-2393. 